Z Rheumatol 2015 · 74:104-105

DOI 10.1007/s00393-014-1448-2

Online publiziert: 19. Februar 2015

c) Springer-Verlag Berlin Heidelberg 2015

A. Zink ${ }^{1} \cdot$ J. Sieper ${ }^{2}$

${ }^{1}$ Deutsches Rheuma-Forschungszentrum (DRFZ), Berlin

2 Med. Klinik I, Rheumatologie, Charité Universitätsmedizin Berlin, Campus Benjamin Franklin

\title{
Von der Ungewissheit zur Evidenz
}

\section{Moderne Statistik in der Rheumatologie}

„Ja, wir werden alles, alles noch einmal in Frage stellen. Und wir werden nicht mit Siebenmeilenstiefeln vorwärtsgehen, sondern im Schneckentempo. Und was wir heute finden, werden wir morgen von der Tafel streichen und erst wieder anschreiben, wenn wir es noch einmal gefunden haben. Und was wir zu finden wünschen, das werden wir, gefunden, mit besonderem Misstrauen ansehen." (Bertold Brecht, Leben des Galilei)

Liebe Kolleginnen und Kollegen,

Galilei spricht hier vom Zweifel als Grundlage jeder wissenschaftlichen Erkenntnis. Die Aufgabe der Wissenschaft ist es nicht, vorgefasste Ansichten zu bestätigen, sondern sie muss ihre mithilfe transparenter Methoden erzielten Ergebnisse selbst immer wieder auf den Prüfstand stellen. In der Medizin bedeutet dieser sich seit dem 19. Jahrhundert vollziehende Paradigmenwechsel die Abkehr von einer durch Autoritäten und Lehrmeinungen geprägten Medizin hin zu einem konsequenten Bezug auf wissenschaftliche Erkenntnisse. Es wird heute von Ärzten selbstverständlich erwartet, dass sie ihre Entscheidungen evidenzbasiert fällen, also aufgrund nachprüfbarer Ergebnisse und nicht allein aus dem eigenen Erfahrungswissen heraus.

\section{$>$ Bei der Gewinnung empirischer Evidenz kommt der Statistik eine herausragende Rolle zu.}

Statistik lehrt uns, dass Wissenschaft nicht auf Gewissheit abzielt, sondern auf den informierten Umgang mit Unsicherheit. Neue Erkenntnisse, die wir durch Experiment oder systematische Beobachtung gewinnen, können unsere vermeintlichen Gewissheiten zunichtemachen.

So objektiv der Umgang mit Zahlen und Ergebnissen auch scheint, so bietet die Wahl der jeweiligen Methoden doch einigen Spielraum, Ergebnisse zu beeinflussen oder unterschiedlich zu interpretieren. Dies gilt sowohl für den Goldstandard der evidenzbasierten Medizin, den randomisierten kontrollierten klinischen Versuch, als auch für nicht randomisierte Studiendesigns. Hier können bewusst (durch einseitige Interessen) oder unbewusst (durch mangelnde Sachkenntnis und unkritische Anwendung von Statistikprogrammen) Ergebnisse erzielt werden, die Nutzen oder Schaden von Therapien größer oder kleiner erscheinen lassen, als diese tatsächlich sind. Neben der wissenschaftlichen Unabhängigkeit der Studiendurchführung und der richtigen Wahl der Fragestellung ist daher die Einbindung statistischer Expertise entscheidend für den Erfolg klinischer Forschung. Für den praktisch tätigen Rheumatologen, der seine Handlungen auf Evidenz gründen soll, ist es entscheidend, dass er Studien beurteilen und in ihrer Glaubwürdigkeit bewerten kann.
Wir haben in diesem Heft fünf (Gruppen von) Experten gebeten, ihre Sicht auf moderne Entwicklungen in der medizinischen Statistik und Studiendurchführung an Beispielen aus der Rheumatologie allgemein verständlich darzustellen.

Der Beitrag von Joachim Gerß, Maria Eveslage, Andreas Faldum und René Schmidt stellt neue Methoden der Durchführung und Analyse randomisierter klinischer Studien (RCT) dar. Unter Gesichtspunkten des Patientenschutzes ebenso wie der hohen Kosten von RCT wurden Methoden entwickelt, die es erlauben, mit immer kleineren Fallzahlen aussagefähige Ergebnisse zu erzielen. Bei der Anwendung adaptiver, sequenzieller Designs wird in vorher festgelegten Zwischenauswertungen das bislang erlangte Wissen für die weitere Studienplanung berücksichtigt. Dadurch können Stichprobenumfänge bei gleicher Aussagefähigkeit verkleinert werden. Neben adaptiven Designs werden zunehmend bayesianisch motivierte statistische Verfahren verwendet, die eine Einbeziehung von Vorwissen aus anderen Analysen bei der Studienplanung und Auswertung erlauben. Adaptive Designs und Bayes-Verfahren sind zudem kombinierbar und werden in Zukunft häufiger zum Einsatz kommen. Wir halten es für sinnvoll, dass Rheumatologen die dahinterliegenden Konzepte kennen.

Der Beitrag von Robert Landewé befasst sich mit modernen Analysemethoden für Beobachtungsstudien. Anders als im randomisierten klinischen Ver- 
such haben wir es in nicht randomisierten Studien immer mit „confounding by indication“ zu tun, also dem systematischen Unterschied zwischen Behandlungsgruppen, der darauf beruht, dass im Alltag Therapien nach klinischer Notwendigkeit und nicht randomisiert vergeben werden. Es gibt unterschiedliche Methoden, diese Verzerrung zu kontrollieren: von der simplen Adjustierung für Unterschiede zwischen den Gruppen bei Beobachtungsbeginn (Baseline-Adjustierung) z. B. mithilfe von Propensity-Scores bis zur longitudinalen, zeitabhängigen Adjustierung um Merkmale, die sich über die Beobachtungszeit hinweg verändern. Welche Methoden jeweils gewählt werden, ist nicht im Einzelnen festgelegt und bedarf sowohl klinischer wie statistischer Expertise.

Wie diese zeitabhängig gewonnenen Ergebnisse aus Beobachtungsstudien dafür genutzt werden können, klinische Entscheidungsprozesse zu unterstützen, macht der Beitrag von Anja Strangfeld und Adrian Richter deutlich. Der RABBIT-Risikoscore ist ein gutes Beispiel dafür, dass sich aus Beobachtungsdaten klinisch relevante Ergebnisse für spezifische Gruppen von Patienten gewinnen lassen, die so niemals aus einem RCT ableitbar wären. Dies liegt sowohl an den kleinen Fallzahlen und der kurzen Beobachtungsdauer im RCT als auch an dem kompletten Fehlen wichtiger Patientengruppen. Ärzte müssen im Alltag mit ganz anderen Patienten umgehen als denjenigen, an denen die Medikamente erprobt wurden. Hierfür sind große Beobachtungsstudien aus der Versorgungsrealität unverzichtbare Informationsquellen.

Dass auch der scheinbar so objektive RCT durchaus in seinen Ergebnissen beeinflussbar ist (z. B. durch Einschlusskriterien, Art der Kontrollgruppe, Interventionsdauer), wird niemanden überraschen. Deshalb ist es von großer Bedeutung, dass es neben den rein Industrieverantworteten RCTs auch sog. „investigator-initiated trials" gibt. Joachim Sieper und Jürgen Braun geben eine Übersicht über 15 Jahre intensiver und erfolgreicher Durchführung klinischer Studien zur axialen Spondyloarthritis. Sie schildern, welche Herausforderungen es bedeutet, ohne die Logistik großer Firmen anspruchsvolle Studien durchzuführen, und wie sich die Bedingungen in den letzten 10 Jahren durch gesetzliche Veränderungen verschärft haben. Sie beschreiben die Voraussetzungen, unter denen auch heute noch Forscher-initiierte klinische Studien - z. B. mit Förderung durch das Bundesministerium für Bildung und Forschung (BMBF)/die Deutsche Forschungsgemeinschaft (DFG) - möglich sind.

In dem abschließenden Beitrag von Rainer Schlittgen wird die Rolle des Statistikers in der klinischen Forschung beleuchtet. Er macht deutlich, wie unverzichtbar statistische Expertise, verbunden mit klinischem Verständnis, für die richtige Auswahl und Interpretation von Methoden ist. Es ist ein zunehmend zu beobachtendes Phänomen, dass man meint, auf qualifizierte Statistiker verzichten zu können zugunsten technisch leicht anwendbarer und vermeintlich anspruchsvoller Statistikprogramme. Um auf Brechts Galilei zurückzukommen: Diese Abkehr von dem Schneckentempo der wissenschaftlichen Genauigkeit hin zu der Verwendung von elektronischen Siebenmeilenstiefeln führt zwar zu einem raschen Anwachsen von Ergebnissen, jedoch bleiben deren Validität und Relevanz oft fraglich.

Alle genannten Autoren haben einen mehr oder weniger engen Bezug zu einem einzigen Statistiker, dem wir diesen Schwerpunkt widmen. Joachim Listing, seit mehr als 20 Jahren leitender Statistiker am Deutschen Rheumaforschungszentrum, feiert am 20.03.2015 seinen 65 . Geburtstag. Er hat an allen beschriebenen klinischen Studien maßgeblich mitgewirkt, die großen Kohortenstudien am Deutschen Rheuma-Forschungszentrum (DRFZ) mit aufgebaut und hat sich international einen Ruf als besonders sorgfältiger und zugleich innovativer Statistiker erworben. Die Arbeitsgruppe am DRFZ, aber auch viele mit ihm kooperierende Rheumatologen sind ihm zu großem Dank verpflichtet. Er hat die klinisch-epidemiologische Forschung in der Rheumatologie entscheidend vorangebracht.
Uns bleibt an dieser Stelle nur zu sagen: Happy Birthday, Joachim, und Danke für alles!

Wir wünschen eine anregende Lektüre.

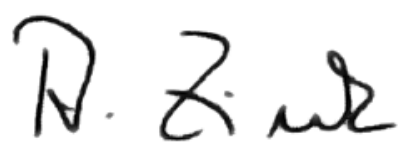

Angela Zink

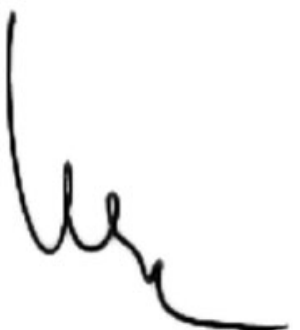

Joachim Sieper

\section{Korrespondenzadressen}

Prof. Dr. A. Zink

Deutsches Rheuma-Forschungszentrum (DRFZ)

Charitéplatz 1, 10117 Berlin

zink@drfz.de

Prof. Dr. J. Sieper

Med. Klinik I, Rheumatologie,

Charité Universitätsmedizin Berlin

Campus Benjamin Franklin

Hindenburgdamm 30, 12200 Berlin

joachim.sieper@charite.de

\section{Einhaltung ethischer Richtlinien}

Interessenkonflikt. A. Zink und J. Sieper geben an, dass kein Interessenkonflikt besteht.

Dieser Beitrag beinhaltet keine Studien an Menschen oder Tieren. 